\title{
Single Atom Imaging and Spectroscopy of Impurities in 2D Materials
}

Wu Zhou ${ }^{1}$, Andrew R. Lupini ${ }^{1}$, Junhao Lin ${ }^{1,2}$, Yongji Gong ${ }^{3}$, Zheng Liu ${ }^{4}$, Myron D. Kapetanakis ${ }^{1,2}$, Mark P. Oxley ${ }^{1}$, Juan-Carlos Idrobo ${ }^{5}$, Stephen J. Pennycook ${ }^{6}$, Sokrates T. Pantelides ${ }^{1,2}$, Pulickel M. Ajayan $^{3}$

${ }^{1 .}$ Materials Science \& Technology Division, Oak Ridge National Laboratory, Oak Ridge, TN 37831, USA

2. Department of Physics and Astronomy, Vanderbilt University, Nashville, TN 37235, USA

${ }^{3 .}$ Department of Chemistry, Department of Materials Science and NanoEngineering, Rice University, Houston, TX 77005, USA

4. School of Materials Science \& Engineering, Nanyang Technological University, 639798, Singapore

5. Center for Nanophase Materials Sciences, Oak Ridge National Laboratory, Oak Ridge, TN 37831, USA

${ }^{6 .}$ Department of Materials Science \& Engineering, National University of Singapore, 117575, Singapore

Impurities have profound influence on the electronic, optical, magnetic, and mechanical properties of materials. Understanding the distribution, dynamics, bonding, and local properties of impurities can, therefore, provide valuable insights into the atomic-scale origins of materials performance. Aberrationcorrected scanning transmission electron microscopy (STEM) at low voltage can now provide real-space imaging and spectroscopy analysis at the atomic scale with single-atom sensitivity. This opens new opportunities for quantitative study of individual impurities in 2D materials. Such studies, especially when combined with first-principles calculations, serve as an important step to correlate the local atomic structure at impurities with local properties, and help to create new functionalities in $2 \mathrm{D}$ materials by controllable doping.

Figure 1 shows that quantitative STEM annular dark field (ADF) image analysis allows us to identify the chemical nature of the dopant and map out their distribution in $\mathrm{MoS}_{2}$ with single atom sensitivity. Such analysis combines computer-assisted atom finding, intensity measurement and site-specific histogram analysis, and statistical analysis for dopant behavior. In bilayer structures, where the preferred stacking generates distinct non-overlapping sites, the dopant distribution can be mapped out one atomiclayer at a time, providing a feasible way to quantify the local composition and measure the local band gap at the $10 \mathrm{~nm}$ scale, as the bandgap is directly related to the local composition [1]. When combined with sequential imaging, such quantitative image analysis can further help to reveal defect dynamics at the single-atom level [2].

Combining the imaging and spectroscopy capabilities of STEM, the changes in local optical response and electronic structure can be directly measured at the single impurity level. As shown in Figure 2, the fine structure in electron energy loss spectra (EELS) provides the sensitivity to determine the nature of the chemical bonding of single atoms. We show that three-dimensional and planar bonding configurations for individual $\mathrm{Si}$ atoms in graphene can be directly discriminated [3]. Furthermore, we show that the presence of a single $\mathrm{Si}$ atom in the graphene lattice can induce local variation to the lowenergy electronic excitations that can be mapped out using valence EELS imaging with atomic resolution $[4,5]$. Recent results on transition-metal doped $\mathrm{MoS}_{2}$ will also be presented [6]. 
References:

[1] Y Gong et al, Nano Lett. 14 (2014), p. 442-449.

[2] J Lin, ST Pantelides and W Zhou, ACS Nano 9 (2015), p. 5189-5197.

[3] W Zhou et al, Phys. Rev. Lett. 109 (2012), p. 206803.

[4] W Zhou et al, Nature Nanotech. 7 (2012), p. 161-165.

[5] MD Kapetanakis et al, submitted (2016).

[6] Research sponsored by the U.S. Department of Energy, Office of Science, Basic Energy Science, Materials Sciences and Engineering Division, and ORNL's Center for Nanophase Materials Sciences (CNMS), which is a DOE Office of Science User Facility.
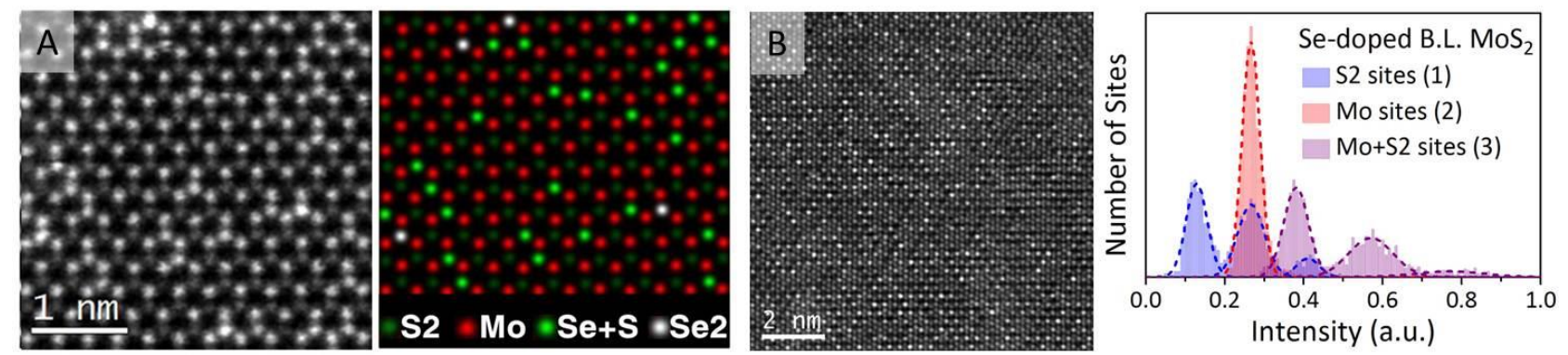

Figure 1. (A) Atom-by-atom Se dopant analysis in monolayer $\mathrm{MoS}_{2}$. (left) Experimental ADF image. (right) Structure model obtained from histogram analysis showing the distribution of single- and doubleSe substituted $\mathrm{S}_{2}$ sites. (B) Layer-by-layer Se dopant analysis in bilayer $\mathrm{MoS}_{2}$. (left) Experimental ADF image of bilayer $\mathrm{Se}-\mathrm{MoS}_{2}$. (right) Site-specific histogram analysis for Se occupation in the top and bottom $\mathrm{MoS}_{2}$ layer. Figures adapted from Ref. [1].
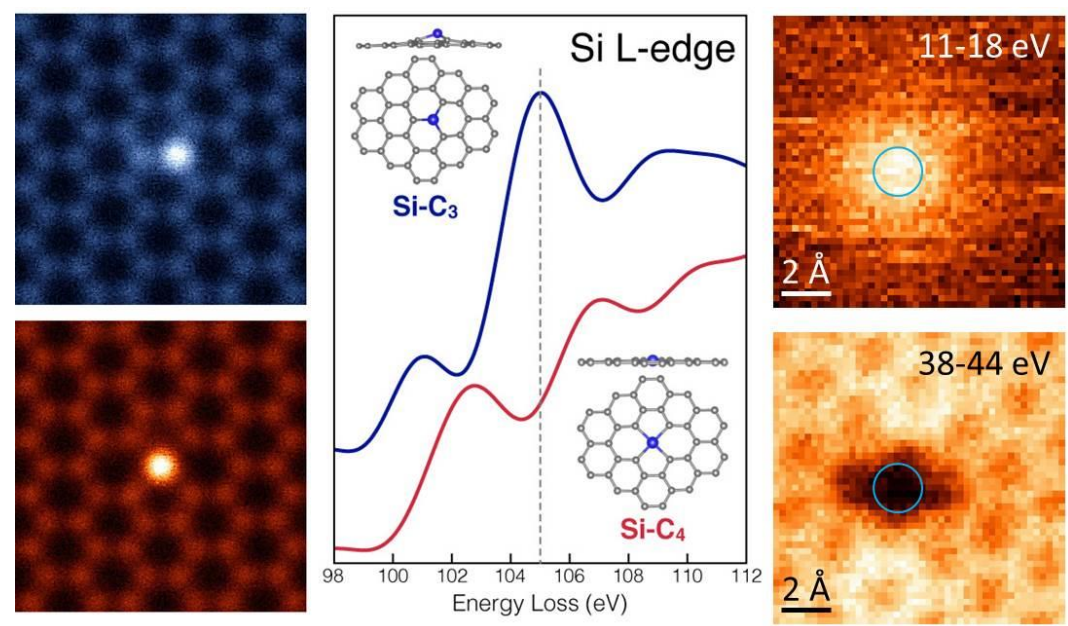

Figure 2. Spectroscopic analysis of single substitutional Si impurities in graphene lattice. (left) ADF images of 3- and 4-fold coordinated Si atoms in graphene; (middle) Si L-edge fine structure extracted from spectrum images of 3- and 4-fold coordinated Si atoms in graphene; (right) spectroscopic maps at different energy-loss regimes of a 4-fold coordinated $\mathrm{Si}$ atom in graphene showing atomic-resolution features. The positions of the Si atoms are indicated by the blue circles. Figures adapted from Refs. [3, 4, $5]$. 